\title{
Role of Pancreatic Stellate Cells in Pancreatic Cancer Metastasis
}

\author{
Zhihong $\mathrm{Xu},{ }^{* \dagger}$ Alain Vonlaufen, ${ }^{* \dagger}$ \\ Phoebe A. Phillips, ${ }^{* \dagger}$ Eva Fiala-Beer, ${ }^{* \dagger}$ \\ Xuguo Zhang, ${ }^{* \dagger}$ Lu Yang, ${ }^{* \dagger}$ Andrew V. Biankin, ${ }^{\mp}$ \\ David Goldstein, ${ }^{\dagger}$ Romano C. Pirola, ${ }^{\dagger}$ \\ Jeremy S. Wilson, ${ }^{* \dagger}$ and Minoti V. Apte ${ }^{\star \dagger}$ \\ From the Pancreatic Research Group,* South Western Sydney \\ Clinical School and School of Medical Sciences, Sydney; the \\ Faculty of Medicine, ${ }^{+}$The University of New South Wales, Sydney; \\ and the Garvan Institute of Medical Research ${ }^{\ddagger}$ Sydney, Australia
}

Pancreatic stellate cells (PSCs) produce the stromal reaction in pancreatic cancer ( $\mathrm{PC}$ ), and their interaction with cancer cells facilitates cancer progression. This study investigated the role of human PSCs (hPSCs) in the metastatic process and tumor angiogenesis using both in vivo (orthotopic model) and in vitro (cultured PSC and PC cells) approaches. A sex mismatch study (injection of male hPSCs plus female PC cells into the pancreas of female mice) was conducted to determine whether hPSCs accompany cancer cells to metastatic sites. Metastatic nodules were examined by fluorescent in situ hybridization for the presence of the Y chromosome. Angiogenesis was assessed by i) immunostaining tumors for $\mathrm{CD} 31$, an endothelial cell marker; and ii) quantifying human microvascular endothelial cell (HMEC-1) tube formation in vitro on exposure to conditioned media from hPSCs. Transendothelial migration was assessed in vitro by examining the movement of fluorescently labeled hPSCs through an endothelial cell monolayer. Human PSCs i) were found in multiple metastatic sites in each mouse injected with male hPSCs plus female PC cells; ii) increased CD31 expression in primary tumors from mice injected with MiaPaCa-2 and hPSCs and stimulated tube formation by HMEC-1 in vitro; and iii) exhibited transendothelial migration that was stimulated by cancer cells. Human PSCs accompany cancer cells to metastatic sites, stimulate angiogenesis, and are able to intravasate/extravasate to and from blood vessels. (Am J Pathol 2010, 177:2585-2596; DOI: 10.2353/ajpath.2010.090899)

Increasing attention has been paid recently to the desmoplastic/stromal reaction in pancreatic cancer and its putative role in cancer progression. Studies in vitro and in vivo have provided strong evidence of the existence of an interaction between pancreatic stellate cells (PSCs, the cells known to produce the stromal reaction in pancreatic cancer) and tumor cells. ${ }^{1-5}$

PSCs are resident cells of the pancreas; in their quiescent (non-activated state) they exhibit abundant vitamin A containing lipid droplets in their cytoplasm. When activated during pancreatic injury, the cells lose their vitamin A stores, assume a myofibroblast-like phenotype, and secrete excessive amounts of the extracellular matrix (ECM) proteins which comprise fibrous tissue. ${ }^{6,7}$

The role of PSCs in pancreatic cancer has been the subject of several studies in recent years. ${ }^{1-5}$ Using an orthotopic model of pancreatic cancer, we have recently shown that mice injected into the pancreas with a suspension of tumor cells mixed with human PSCs (hPSCs) develop larger tumors with extensive desmoplasia and also exhibit increased regional and distant spread, compared to mice injected with tumor cells alone. ${ }^{5}$ In vitro studies have established that pancreatic cancer cells induce proliferation, migration, and extracellular matrix production by PSCs. ${ }^{2,5}$ In turn, PSCs increase pancreatic cancer cell migration and proliferation, but at the same time, decrease cancer cell apoptosis, thereby enhancing the survival of cancer cells. ${ }^{4,5}$ These observations support the concept that pancreatic cancer cells recruit stromal cells to produce a growth permissive environment that facilitates cancer progression. ${ }^{8}$

The dismal prognosis of pancreatic cancer is thought to be related to its propensity for early lymphatic and

Supported by grants from The National Health and Medical Research Council of Australia (350838) and the Cancer Council of New South Wales, Australia (RG 08-05). M.A., A.B., and D.G. are principal investigators on a STREP grant from the Cancer Council of New South Wales which has supported the establishment of a pancreatic cancer tumor bank and database - the NSW Pancreatic Cancer Network (NSWPCN).

Accepted for publication June 29, 2010

Supplemental material for this article can be found on http://ajp. amjpathol.org.

Current address for A.V.: Department of Gastroenterology and Hepatology, University Hospital, Geneva, Switzerland.

Address reprint requests to Professor Minoti V. Apte, Ph.D., Director, Pancreatic Research Group, Faculty of Medicine, Room 505, Level 5, Wallace Wurth Building, The University of New South Wales, Sydney, NSW 2052, Australia. E-mail: m.apte@unsw.edu.au. 
hematogenous spread. Most patients exhibit evidence of extra-pancreatic dissemination at diagnosis, and their fiveyear survival rate is a low $2 \%$ compared to the $20 \%$ five-year survival of patients with localized pancreatic tumors. ${ }^{9}$ In general, cancer cell metastasis involves loss of cell-cell adhesion, increased motility/migration, intravasation into blood and/or lymph vessels, transport through the circulation, extravasation, and finally seeding at distant sites. ${ }^{10,11}$ We have previously shown that PSCs can stimulate motility/migration of cancer cells in vitro, ${ }^{5}$ suggesting that PSCs may facilitate at least one of the above-mentioned steps in the metastatic process. There has been an interesting shift in recent years in our concept of the way metastatic cells may travel within the blood or lymphatic circulation, with increasing recognition of the fact that metastatic cancer cells travel through the circulation not only as single cells but also as cell clusters. ${ }^{11-15}$ Given the close proximity of PSCs to cancer cells, it would not be unreasonable to postulate that PSCs may accompany cancer cells to distant metastatic sites as part of such metastatic cell clusters.

A central process in cancer metastasis and local tumor growth is the formation of new blood vessels (neoangiogenesis). Among the most prominent of several recently identified proangiogenic factors is vascular endothelial growth factor (VEGF) ${ }^{16,17}$; up-regulation of VEGF expression in tumor tissue has been reported to be associated with poor prognosis in several cancers including pancreas. ${ }^{18-25}$ In pancreatic cancer, VEGF has been reported to be produced not only by pancreatic cancer cells but also by PSCs. ${ }^{26}$ Other known proangiogenic growth factors include platelet-derived growth factor (PDGF, known to be produced by PSCs), 27 and hepatocyte growth factor (HGF) and fibroblast growth factor which are up-regulated in the stroma of pancreatic cancer. ${ }^{28,29}$ Thus, it is possible that PSCs play a role in angiogenesis in pancreatic cancer.

To date, interactions between PSCs and pancreatic cancer cells have been examined mainly using human PSCs isolated by the outgrowth method from pancreatic tissue resected from patients with pancreatic cancer. ${ }^{2,4,5}$ As such, these PSCs would be reasonably assumed to have been in contact with and under the influence of cancer cells for some time before their use in experiments. Thus, these cells would be expected to be 'primed' by the cancer cells in vivo. Little is known about the mechanisms that mediate the evolution of PSCs from their normal phenotype to a cancer-associated phenotype in vivo, nor is it known whether 'normal' PSCs can play a similarly facilitatory role in pancreatic cancer as has been demonstrated with cancer-associated hPSCs.

The present study was designed to determine whether PSCs (both cancer-associated and normal) i) accompany cancer cells to distant metastatic sites; ii) play a role in neoangiogenesis in pancreatic cancer; and iii) have the capacity to migrate through an endothelial lining.

\section{Materials and Methods}

\section{Reagents}

The reagents included Iscove's Modified Dulbecco's Medium, RPMI Medium 1640, MCDB 131 medium, fetal bovine serum, glutamine, and $4^{\prime}, 6$-diamidino-2-phenylindole (DAPI) from Invitrogen (Mulgrave, VIC, Australia); Dulbecco's Modified Eagle's Medium and Xten Hybricell SFM Medium from Thermo Fisher Scientific (Waltham, $\mathrm{MA}$ ); anti- $\alpha$-smooth muscle actin ( $\alpha \mathrm{SMA}$ ) antibody, Ribonuclease A, Proteinase K, and formamide from Sigma (St. Louis, MO); anti-cytokeratin antibody, anti-proliferating cell nuclear antigen (PCNA) antibody and 3,3'-diaminobenzidine (DAB) tetrahydrochloride substrate from DAKO (Campbellfield, VIC, Australia); anti-CD31 antibody and basement membrane matrix growth factor reduced Matrigel from BD Bioscience (North Ryde, NSW, Australia); anti-glial fibrillary acidic protein (GFAP) antibody and cell culture inserts with 8-micron pores from Millipore (North Ryde, NSW, Australia); fluorescent centromeric probe for the $\mathrm{Y}$ chromosome from Cambio (Cambridge, UK); Safil 5/0 Polyglycolic acid absorbable surgical suture from B. Braun (Bella Vista, NSW, Australia); CytoSelect Tumor Transendothelial Migration Assay kit from Cell Biolabs, Inc. (San Diego, CA).

These studies were approved by the Human Research Ethics Committee and the Animal Care and Ethics Committee of The University of New South Wales, Sydney, Australia.

\section{Cell Culture}

Human pancreatic cancer cell lines MiaPaCa-2, AsPC-1 (American Type Culture Collection, Manassas, VA) and human microvascular endothelial cells (HMEC-1, a kind gift from Professor Philip Hogg, UNSW Cancer Research Centre, Sydney, Australia) were cultured according to supplier's instructions and published protocols. ${ }^{30}$

\section{Human PSCs}

Cancer-associated hPSCs (CAhPSCs) were isolated from pancreatic tissue obtained from pancreatic cancer patients undergoing resection, using the outgrowth method. ${ }^{31}$ Normal hPSCs (NhPSCs) were isolated using a density gradient isolation method from the histologically normal portion of resected pancreatic tissue from patients with benign conditions of the pancreas. ${ }^{32}$ The purity of hPSC yield was assessed by morphology and immunostaining for the selective marker GFAP and the activation marker $\alpha$ SMA. ${ }^{32}$

\section{Orthotopic Model of Pancreatic Cancer}

Because we aimed to use two types of hPSCs (normal and cancer-associated), our experiments included two separate batches. For both batches, eight-week-old female athymic mice (BALB/c nu/nu) were assigned to sets consisting of three mice each. Each set of mice was operated on the one occasion and injected with cells from the same cell preparations (hPSC and pancreatic cancer cell line); thus within a set, direct comparisons could be made between the mice without any confounding variability in the types/source of cells injected into the pancreas. The surgery was performed as described in 
our previous publication. ${ }^{5}$ An incision was made in the left flank, and a portion of the spleen and tail of pancreas was exteriorized. Mice in each set were injected into the tail of the pancreas with one of the following: i) AsPC-1 cells $1 \times 10^{6}$; ii) AsPC-1 + normal or cancer-associated hPSCs $1 \times 10^{6}$ each; iii) normal or cancer-associated hPSCs $1 \times 10^{6}$. The injection site was gently pressed immediately with betadine-soaked gauze for 30 seconds, before returning the pancreas and spleen to the abdomen. There were $n=14$ mice per group for experiments using CAhPSCs and $n=8$ mice per group for studies using NhPSCs.

Mice were sacrificed six weeks after operation. Pancreatic tumor size was measured as described previously. ${ }^{5}$ Metastatic lesions in abdominal and thoracic cavities were identified and relevant pieces of tissue collected. Paraffin sections of primary tumors and pancreas from control mice injected with hPSCs alone were stained using H\&E and Sirius Red. Sections were also immunostained for $\alpha$ SMA, cytokeratin, and PCNA.

Sections of tissues carrying metastatic nodules were histopathologically assessed. Selected liver metastatic nodules were immunostained for $\alpha$ SMA and PCNA to identify activated stellate cells and proliferating cancer cells.

\section{Immunostaining for $\alpha S M A$, Cytokeratin, and PCNA in Primary Tumors and Metastatic Nodules}

Immunostaining and morphometric analyses for $\alpha \mathrm{SMA}$ and cytokeratin were performed as described by us previously. ${ }^{5}$ PCNA staining was also performed as published previously ${ }^{5}$ and assessed by a "grid point counting" method which involved counting of cells of interest on photomicrographs that were overlaid by a grid comprising 117 evenly distributed points of intersection ("grid points"). Only the PCNA-positive cells that coincided with a grid point were counted and expressed as a percentage of the total 117 grid points. To further confirm that these PCNA-positive cells were cancer cells, we performed additional immunostaining (for PCNA and cytokeratin) studies of serial sections of primary tumors from mice injected with AsPC-1 alone or AsPC-1 + hPSCs. Staining was assessed by grid point counting using the grid on the Aperio ImageScope system which comprises 441 evenly distributed grid points. This imaging system allows accurate matching of the orientation and magnification of serial sections such that the grid points fall on exactly the same points of the two serial sections. In five randomly selected matching high-power fields $(\times 200)$ of serial section pairs, cytokeratin-positive cells that were also PCNA-positive were counted. Data are expressed as percentage of cancer cells (cytokeratin-positive) that are also PCNA-positive, thus providing an indication of the fraction of proliferating cancer cells in each tumor.

\section{Sex Mismatch Study}

\section{Fluorescent in Situ Hybridization (FISH)}

To determine whether hPSCs were able to accompany pancreatic cancer cells to metastatic sites, a sex mismatch study was performed using female mice implanted into the pancreas with female cancer cells \pm male hPSCs. The presence of the $Y$ chromosome in metastatic nodules would confirm the presence of exogenously introduced male hPSCs. Y chromosomes were detected using a Cy3-labeled fluorescent probe (Cambio, Cambridge, UK), which binds to the centromere of the $Y$ chromosome; this was applied according to the manufacturer's protocol, with minor modifications. Briefly, 5-micron cryosections of metastatic nodules were fixed with $4 \%$ paraformaldehyde, deproteinized with $0.2 \mathrm{~N} \mathrm{HCl}$, and permeabilized with acetone. Then, cryosections were treated with RNase A $100 \mu \mathrm{g} / \mathrm{ml}$ at $37^{\circ} \mathrm{C}$ for 1 hour followed by proteinase $\mathrm{K} 10 \mu \mathrm{g} / \mathrm{ml}$ at $37^{\circ} \mathrm{C}$ for 30 minutes. DNA was denatured with $70 \%$ formamide at $70^{\circ} \mathrm{C}$ for 2 minutes and hybridized at $37^{\circ} \mathrm{C}$ overnight with the Cy3labeled fluorescent probe (Red). Posthybridization wash at $37^{\circ} \mathrm{C}$ comprised $50 \%$ formamide for 5 minutes $\times 2$ and $2 \times$ SSC for 5 minutes $\times 2$. Sections were incubated with DAPI for nuclear staining (blue) and imaged by confocal microscope Olympus FV1000. All other reagents were from Sigma-Aldrich unless specified.

\section{FISH for Y Chromosome and Immunostaining for Cytokeratin on Serial Sections of Metastatic Nodules}

Serial cryosections of metastatic nodules in liver, diaphragm, and mediastinum were stained for the $Y$ chromosome (FISH) or cytokeratin (immunohistochemistry). The total number of $Y$ chromosome-positive cells or cytokeratin-positive cells on serial sections was counted. Data were expressed as the number of $Y$ chromosomepositive cells per 100 cytokeratin-positive cells in the same nodule.

\section{Dual Staining for Y Chromosome + GFAP or $\alpha$ SMA}

FISH was performed as described above except proteinase $\mathrm{K}$ was used at a concentration of $5 \mu \mathrm{g} / \mathrm{ml}$ at $37^{\circ} \mathrm{C}$ for 15 minutes. This was followed by immunostaining for GFAP or $\alpha S M A$, wherein sections were incubated at $4^{\circ} \mathrm{C}$ overnight with primary antibody for GFAP (1:100) or $\alpha$ SMA (1:200). $\lg G_{1}$ or $\operatorname{lgG}_{2 a}$ respectively were applied as negative control. Goat anti-mouse secondary antibody labeled with Alexa-Fluor ${ }^{488}$ (Green, 1:1000, Invitrogen) was applied at room temperature for 30 minutes, followed by DAPI staining.

\section{Angiogenesis}

\section{In Vivo}

Endothelial cell numbers in primary tumors were assessed by immunostaining for the endothelial cell marker CD31. Tumors produced by injection of MiaPaCa-2 \pm hPSCs (from our recently published model ${ }^{5}$ ) and those 
A
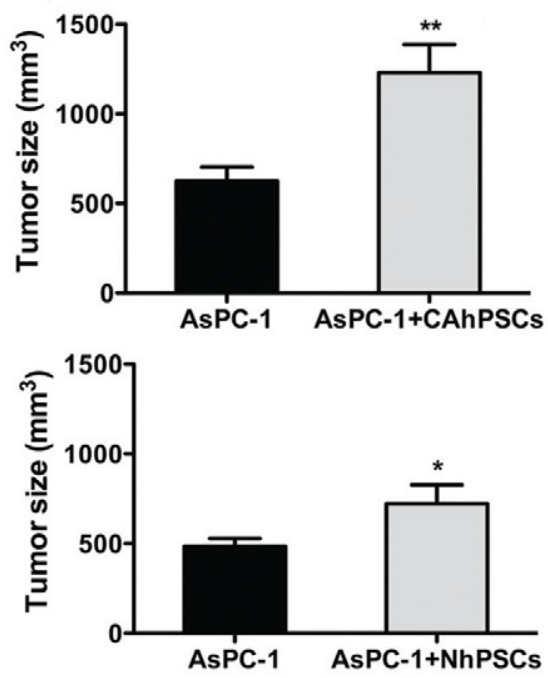

C
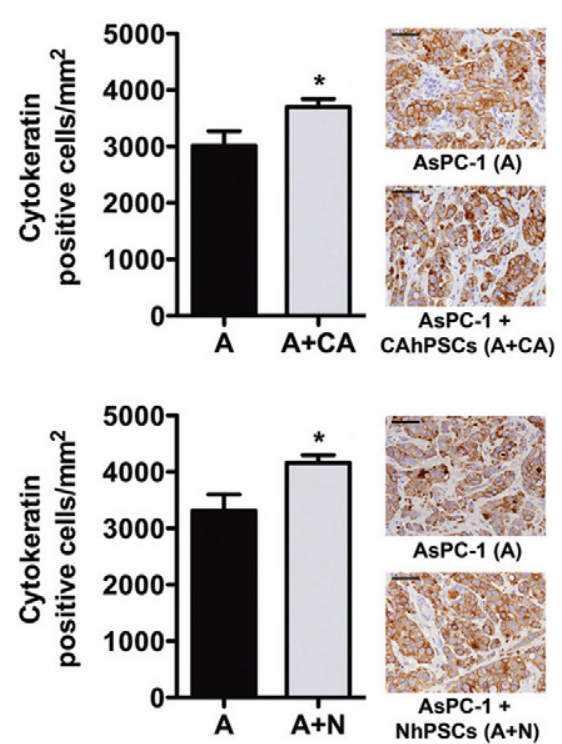

B


D
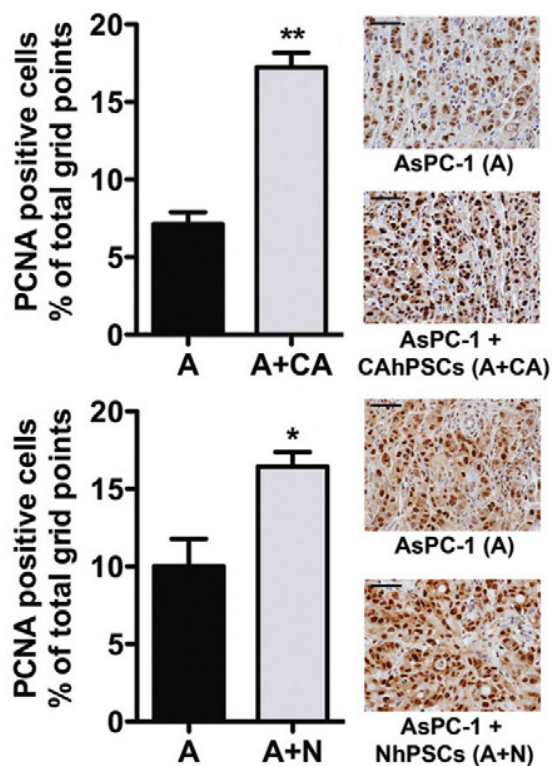

Figure 1. Primary tumors in orthotopic model A: Tumor size: Primary tumors in mice injected with AsPC-1 + CAhPSCs were significantly larger than tumors in mice injected with AsPC-1 alone $(* * P<0.005 ; n=14$ per group). Similar results were observed in mice injected with AsPC- $1 \pm$ NhPSCs (*P $<0.05 ; n=8$ per group). B: Fibrosis in primary tumors: Representative Sirius Red-stained images $(\times 400)$ showing significantly increased collagen deposition in primary tumors in the presence of CAhPSCs $(* * P<$ $0.001 ; n=14$ per group) and NhPSCs $\left({ }^{*} P<0.05\right.$; $n=8$ per group). C: Density of cancer cells: Representative images $(\times 600)$ of sections immunostained for cytokeratin. The density of cytokeratin-positive cells was significantly higher in the primary tumors from mice injected with AsPC-1 + CAhPSCs or NhPSCs compared to tumors in mice injected with AsPC- 1 alone $\left({ }^{*} P<\right.$ 0.05; $n=14$ per group and $n=8$ per group, respectively). D: Cell proliferation in primary tumors: Immunostaining $(\times 600)$ for PCNA, showing that the proportion of PCNA-positive cells was significantly increased in primary tumors from mice injected with AsPC-1 + CAhPSCs (** $P<0.0001 ; n=14$ per group) compared with that in tumors from mice injected with AsPC-1 alone. Similar results were found in mice injected with AsPC- $1 \pm$ NhPSCs $\left({ }^{*} P<0.05 ; n=8\right.$ per group). Scale bars $=50 \mu \mathrm{m}$. produced by AsPC- $1 \pm$ hPSCs (in the current study) were examined. Acetone fixed cryosections (5-micron) were incubated with rat anti-mouse CD31 antibody (1:50, BD Bioscience) at room temperature for 1 hour then with a biotinylated polyclonal goat anti-rat secondary antibody (1:50, BD Bioscience) at room temperature for 30 minutes. Subsequently, sections were incubated with Streptavidin HRP for 30 minutes, then with DAB substrate, and morphometrically analyzed.

\section{In Vitro}

To assess the effect of hPSC-derived secretions on angiogenesis in vitro and to determine whether any observed effect was mediated via VEGF, a tube formation assay was performed. HMEC-1 cells seeded on 96-well plates coated with Matrigel (growth factor reduced basement membrane matrix, BD Bioscience, Australia), ${ }^{33,34}$ were treated in triplicate for 6 hours at $37^{\circ} \mathrm{C}$ with conditioned-media from different hPSC preparations. For VEGF experiments, conditioned media from hPSCs were preincubated with $2 \mu \mathrm{g} / \mathrm{ml}$ anti-human VEGF neutralizing antibody or equivalent concentration of $\operatorname{lgG}_{2 b}$ at $37^{\circ} \mathrm{C}$ for 1 hour. Four high-power images per well were taken using a Nikon Eclipse TE2000-S microscope with a Photometrics Cool SNAP ES still camera. The number of closed tube structures formed by HMEC-1 cells was counted.

\section{Transendothelial Migration}

A transendothelial migration assay using the CytoSelect ${ }^{\mathrm{TM}}$ Tumor Transendothelial Migration Assay kit (Cell Biolabs, 
Table 1. Incidence of Metastasis in Orthotopic Model

\begin{tabular}{lcccc}
\hline \multicolumn{5}{c}{ Incidence of metastasis (Number of animals with metastasis) } \\
\hline Metastatic sites & $\begin{array}{c}\text { AsPC-1 } \\
(n=14)\end{array}$ & $\begin{array}{c}\text { AsPC-1 }+ \text { CAhPSCs } \\
(n=14)\end{array}$ & $\begin{array}{c}\text { AsPC-1 } \\
(n=8)\end{array}$ & $\begin{array}{c}\text { AsPC-1 }+ \text { NhPSCs } \\
(n=8)\end{array}$ \\
\hline Liver & 5 & 12 & 3 & 7 \\
Mesentery & 3 & 8 & 3 & 5 \\
Kidney & 0 & 7 & 1 & 4 \\
Diaphragm & 1 & 5 & 1 & 4 \\
Mediastinum & 3 & 6 & 0 & 3 \\
Lung & 0 & 0 & & 1 \\
\hline
\end{tabular}

The incidence of metastasis to several different sites increased significantly in mice injected with AsPC-1 + CAhPSCs compared with that in mice injected with AsPC-1 alone $\left({ }^{*} P<0.0001\right)$. Similarly, the incidence of metastasis was significantly greater in the presence of NhPSCs than that in mice injected with AsPC-1 alone ( $\left.{ }^{\dagger} P=0.001\right)$. Data were analyzed by two-tailed Fisher's exact test.

Inc., San Diego, USA) was performed according to the manufacturer's instructions. Briefly, a monolayer of HMEC-1 cells on porous inserts $(8 \mu \mathrm{m})$ in 24-well plates was treated with $10 \mathrm{ng} / \mathrm{ml} \mathrm{TNF} \alpha$ at $37^{\circ} \mathrm{C}$ for 4 hours to activate endothelial cells and induce relocation of adhesion molecules. ${ }^{35}$ Human PSCs were labeled with CytoTracker ${ }^{\mathrm{TM}}$ (fluorescence). 150,000 labeled hPSCs were mixed with 150,000 unlabeled hPSCs or unlabeled AsPC-1 cells and placed over the endothelial monolayer. After 48 hours, nonmigratory cells were removed by cotton-tipped swabs from the top of the inserts, and the inserts were transferred into a well containing $200 \mu$ l lysis buffer. After incubation, $100 \mu \mathrm{l}$ samples were measured using SpectraMax M2 microplate reader $(480 \mathrm{~nm} / 520 \mathrm{~nm}$, Molecular Devices, Sunnyvale, CA).

To determine whether any effect of cancer cells on CAhPSC transendothelial migration was mediated by PDGF, conditioned medium from AsPC-1 cells was preincubated with $50 \mu \mathrm{g} / \mathrm{ml}$ anti-human PDGF-BB neutralizing antibody or equivalent concentration of goat $\operatorname{lgG}$ at $37^{\circ} \mathrm{C}$ for 1 hour. Transendothelial migration of CAhPSCs was then assessed after 48 hours incubation with treated or untreated AsPC-1 conditioned medium at $37^{\circ} \mathrm{C}$.

\section{Statistical Analysis}

Data are expressed as means \pm SE. Student's t-test, one-way analysis of variance with Tukey's post hoc test, or Fisher's exact test was applied as appropriate. ${ }^{36}$

\section{Results}

Given the known heterogeneity of pancreatic cancer, the orthotopic model in this study involved the use of AsPC-1 (a poorly differentiated pancreatic cancer cell line established from ascitic fluid of a female patient with PC) as against the moderately differentiated cell line (MiaPaCa-2) used in our recent publication. ${ }^{5}$

\section{Primary Tumor Characteristics}

In general, palpable tumors developed earlier in mice injected with AsPC-1 + hPSCs compared to mice injected with AsPC-1 alone. Macroscopically, tumors appeared as a firm, white, single, irregular mass; some larger tumors exhibited central necrosis. Tumors in mice injected with AsPC1 + CAhPSCs or AsPC-1 + NhPSCs were significantly larger than those in corresponding controls (mice injected with AsPC-1 alone; Figure 1A). Importantly, no tumors were found (macroscopically or microscopically) in mice injected with hPSCs (cancer-associated or normal) alone, confirming that PSCs themselves do not have tumorigenic potential.

The orthotopic nature of tumors in this model was confirmed by H\&E sections of primary tumors (Please see Supplemental Figure S1 at http://ajp.amjpathol.org). Obvious bands of fibrosis were seen in primary tumors from mice injected with cancer cells + hPSCs, resembling the desmoplasia of human pancreatic cancer. These tumors also exhibited significantly increased collagen deposition, cytokeratin positivity, and PCNA staining (Figure 1, $\mathrm{B}-\mathrm{D}$ ). The proportion of proliferating cancer cells (cytokeratin-positive cells that were also PCNA-positive) was as follows: AsPC- 1 alone versus AsPC- $1+$ CAhPSCs, $39.51 \pm 1.90 \%$ versus $47.70 \pm 2.58 \%, P<0.01 n=8$; AsPC- 1 alone versus AsPC- $1+$ NhPSCs, $38.14 \pm 1.45 \%$ versus $49.08 \pm 1.28 \%, P<0.005 n=7$. These results

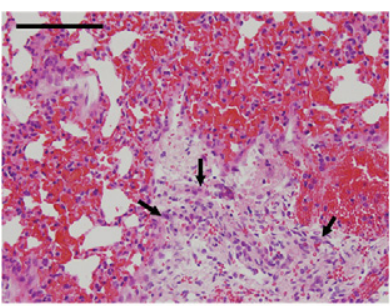

Metastasis to lung



Metastasis to liver

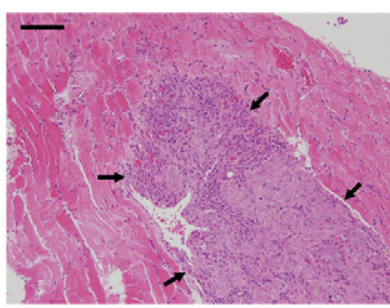

Metastasis to diaphragm

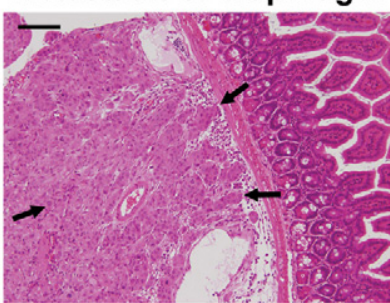

Metastasis to mesentery
Figure 2. Representative photomicrographs of metastatic nodules. H\&Estained sections of nodules (demarcated by arrows) in lung $(\times 400)$, diaphragm $(\times 200)$, liver $(\times 200)$, and mesentery $(\times 200)$. Scale bars $=100 \mu \mathrm{m}$. 


\section{A FISH for y chromosome}
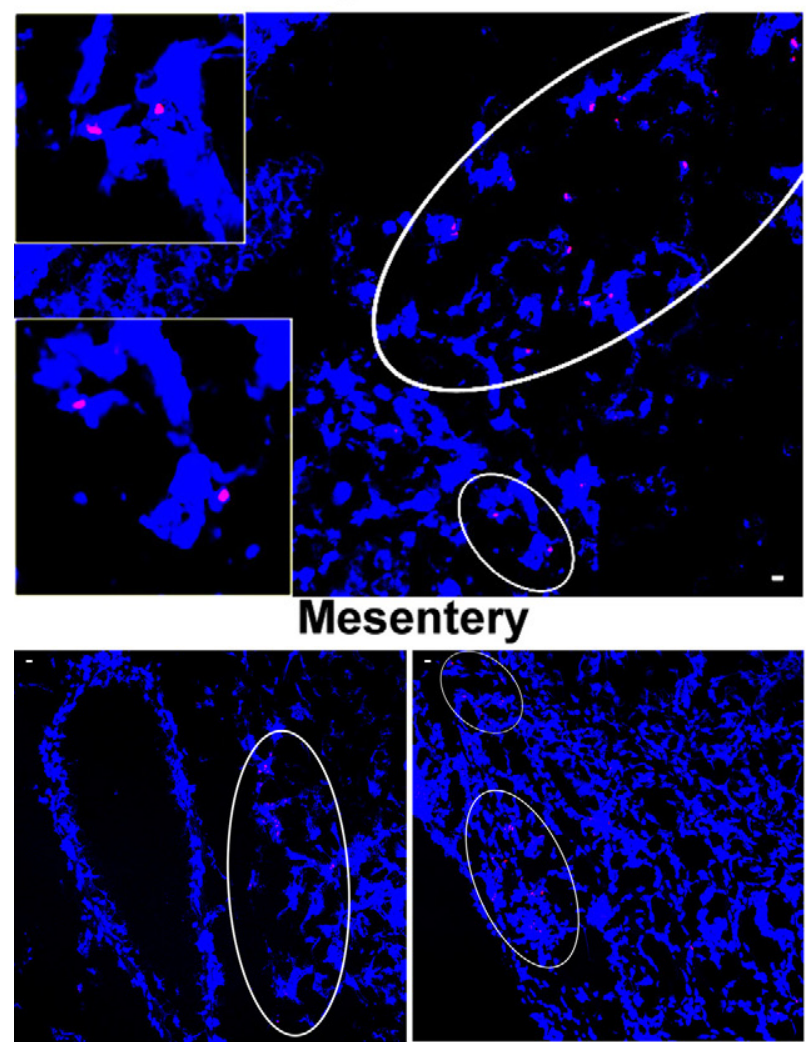

Liver

C

FISH Positive

AsPC-1 + male NhPSCs

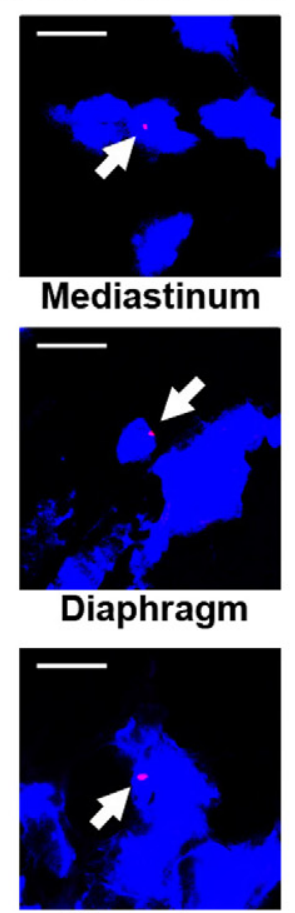

Mesentery

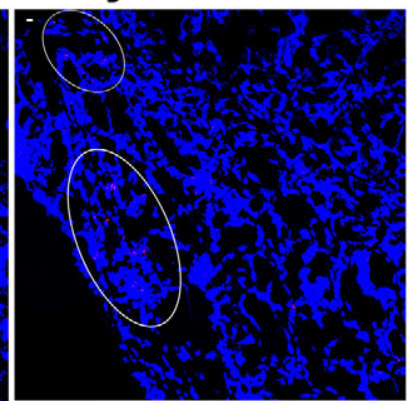

Diaphragm

FISH Negative AsPC-1 alone

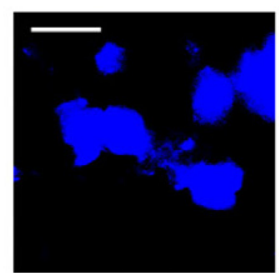

Mediastinum

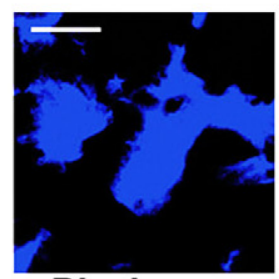

Diaphragm

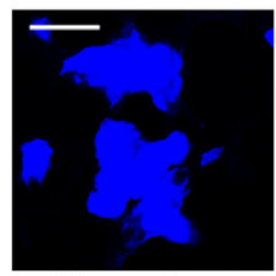

Mesentery

B

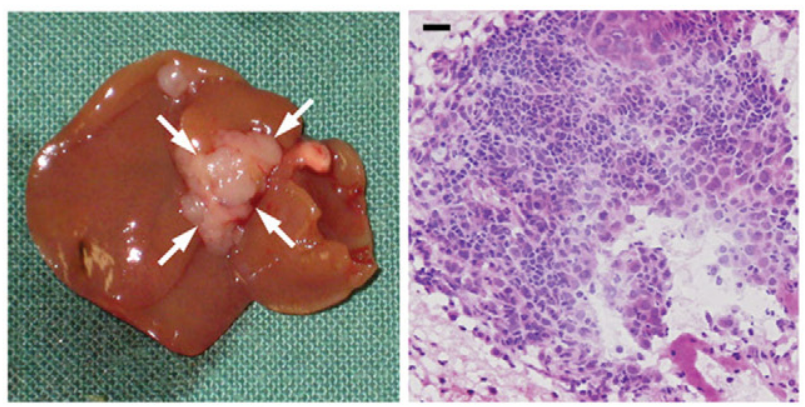

Liver metastatic nodule

H\&E of liver nodule

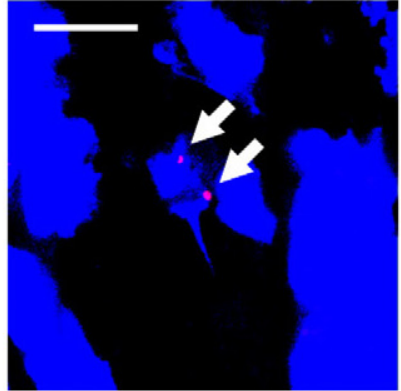

FISH positive AsPC-1 + male CAhPSCs

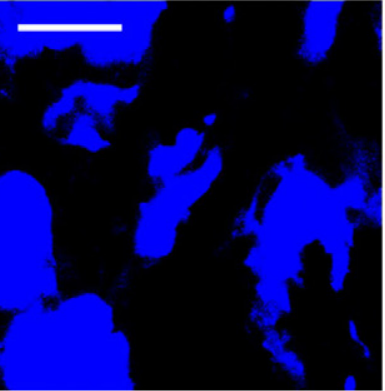

FISH negative AsPC-1 alone

D

FISH + GFAP/ $\alpha$ SMA staining (AsPC-1 + male hPSCs)



Diaphragm
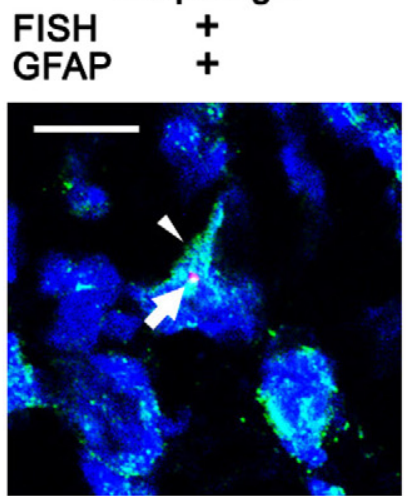

Mediastinum

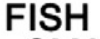
$\alpha$ SMA

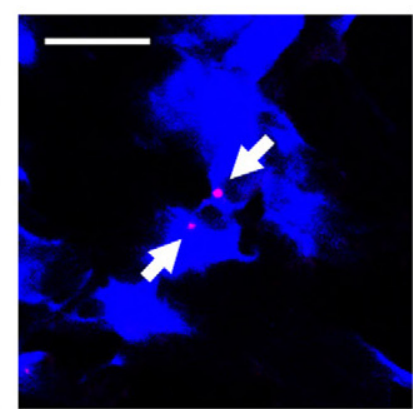

Diaphragm

$+$

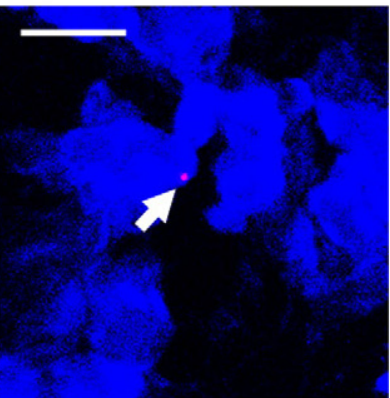

Mediastinum

$+$ 
indicate that the observed increase of tumor size in the presence of hPSCs was due to both PSC-induced fibrosis and increased cancer cell proliferation. [Note: The increase in tumor size in AsPC-1 + hPSC injected mice is not merely a reflection of the difference in the number of cells initially injected into the pancreas in the two groups (ie, 1 million cancer cells for the AsPC-1 alone group and 1 million cancer cells +1 million stellate cells for the AsPC-1+hPSC group). Our studies using an equal number of cells in the two groups ie, 1 million cancer cells in AsPC- 1 alone group and 0.5 million cancer cells +0.5 million stellate cells (total of 1 million cells) in the AsPC$1+$ hPSC group, have shown that despite a halving of the initial tumorigenic potential (from AsPC-1 cells) in the latter group, the tumor volumes in the two groups were similar. In addition, quantitative analysis of cytokeratin stained cells in primary tumors showed that the number of cancer cells per $\mathrm{mm}^{2}$ in AsPC-1 + hPSC tumors was higher than that in AsPC-1 alone tumors (4143 \pm 187.9 versus $3231 \pm 171.7$ per $\mathrm{mm}^{2}$ respectively; $\mathrm{P}<0.02 ; n=$ 6 animals per group). Furthermore, the incidence of regional and distant metastasis was also higher in mice injected with AsPC-1 + hPSC compared to mice injected with AsPC-1 alone. Please see Supplemental Tables 1 and 2 at http://ajp.amjpathol.org.

\section{Proportion of Activated PSCs in Primary Tumors}

Assessment of serial sections of AsPC-1 + hPSC tumors for $\alpha$ SMA-positive cells (activated PSCs) and cytokeratinpositive cells (cancer cells) revealed that there were $15.33 \pm 1.24$ activated PSCs per 100 cytokeratin positive cells (tumor cells) in the section.

\section{Characteristics of Metastatic Nodules}

The incidence of regional and distant metastasis was significantly higher in mice injected with AsPC-1 + hPSCs (cancer-associated or normal) than that in mice receiving AsPC-1 alone (Table 1). Metastatic nodules were found in

\footnotetext{
Figure 3. Identification of male hPSCs in metastatic nodules. A: FISH for the Y chromosome: Representative photomicrographs $(\times 200)$ showing Y chromosome-positive cells in metastatic nodules in the mesentery (insets are high-power views of the circled regions), liver, and diaphragm from mice injected with AsPC-1 + male hPSCs. B: Macroscopic appearance of a representative liver metastatic nodule from a mouse injected with AsPC-1 + male CAhPSCs and a photomicrograph $(\times 400)$ of an H\&E-stained section of a liver nodule. Lower panels show representative photomicrographs $(\times 1000)$ of FISH for the $\mathrm{Y}$ chromosome (positive pink staining; arrows) in a liver metastatic nodule from a mouse injected with AsPC-1 + male CAhPSCs and no staining for the $\mathrm{Y}$ chromosome in a liver nodule from a mouse injected with AsPC-1 alone. C: FISH for the Y chromosome: Representative photomicrographs $(\times 1000)$ showing positive pink staining (arrows) for the $\mathrm{Y}$ chromosome in metastatic nodules from mice injected with AsPC-1 + male NhPSCs (mediastinum, diaphragm, and mesentery). No such staining was observed in metastatic nodules of mice injected with AsPC-1 cells alone. D: Dual staining of cryosections: Representative photomicrographs of metastatic nodules stained for the Y chromosome using FISH (pink; arrows) and i) the PSC-selective marker GFAP (green; arrowheads) from mice injected with AsPC- $1+$ male NhPSCs or ii) the PSC activation marker $\alpha$ SMA (green; fine arrowhead) from mice injected with AsPC-1 + male CAhPSCs. Also shown are the corresponding negative controls for GFAP and $\alpha \mathrm{SMA}$ where the FISH-positive sections were incubated with $\operatorname{IgG}_{1}$ or $\operatorname{IgG}_{2 \mathrm{a}}$ respectively. Scale bars $=10 \mu \mathrm{m}$.
}

the mesentery, liver hilum, kidney, diaphragm, mediastinum, and lung (Figure 2).

Compared to liver nodules from mice injected with AsPC-1 alone, those from mice injected with AsPC-1 + hPSCs exhibited no change in PCNA staining (data expressed as \% of total grid points, $17.9 \pm 1.3$ versus $19.3 \pm 1.9 ; n=4$ per group), but a significant increase in $\alpha$ SMA expression (data expressed as \% of total area, $7.8 \pm 2.1$ versus $13.4 \pm 2.7, P<0.05 n=4$ per group); increased $\alpha$ SMA suggests the presence of increased numbers of activated stellate cells (which may include exogenous hPSCs as well as mouse hepatic stellate cells recruited within the liver; see Discussion).

\section{Presence of Human PSCs from Primary Tumor in Metastatic Nodules}

Positive $Y$ chromosome staining (pink) representing exogenously introduced male hPSCs, was observed in multiple metastatic sites (including mediastinum, liver, diaphragm, mesentery) in each mouse injected with AsPC-1 + male hPSCs (cancer-associated and normal). [Note: FISH involves significant protease digestion of tissue to obtain adequate exposure of chromosomes to the probe. This can result in disruption of cellular architecture]. Figure 3A depicts positive staining for $Y$ chromosomes in the metastatic nodules in mesentery, liver, and diaphragm from mice injected with AsPC-1 + male hPSCs. Figure 3B depicts i) a photograph of a liver metastatic nodule from a mouse injected with AsPC-1 + CAhPSCs; ii) sections of the liver nodule subjected to $\mathrm{H} \& \mathrm{E}$ staining and FISH for the $\mathrm{Y}$ chromosome (pink); and iii) a liver metastatic nodule from a mouse receiving AsPC-1 alone showing no $\mathrm{Y}$ chromosome staining as was to be expected. Figure $3 \mathrm{C}$ shows FISH-positive staining in metastatic nodules in the diaphragm, mesentery, and mediastinum from mice injected with AsPC-1 + male NhPSC. Importantly, the Y chromosome-positive cells also expressed the stellate cell identifiers GFAP and $\alpha$ SMA (Figure 3D), confirming that they were PSCs. Z-stack images (confirming $Y$ chromosome positive staining in the nucleus) have also been provided as a Supplemental video at http://ajp.amjpathol.org.

\section{Proportion of Human PSCs in Metastatic Nodules}

Cell counts from selected metastatic nodules from mice injected with AsPC-1 + male hPSCs stained for FISH and cytokeratin indicated that the number of male hPSCs per 100 cytokeratin-stained cells (tumor cells) was $5.62 \pm$ 1.81 (Table 2). Data for individual metastatic sites are also noted in Table 2.

\section{Effect of Human PSCs on Angiogenesis}

\section{In Vivo}

Morphometric analysis of CD31 stained cryosections of primary tumors from our recently published mode 
Table 2. Quantification of hPSCs in Metastatic Sites

\begin{tabular}{lc}
\hline \multicolumn{1}{c}{ Metastatic sites } & $\begin{array}{c}\text { Number of male hPSCs } \\
\text { expressed as proportion of } \\
\text { cancer cells (mean } \pm \text { SE) }\end{array}$ \\
\hline Liver $(n=5)$ & $5.78 \pm 2.93 \%$ \\
Diaphragm $(n=3)$ & $6.14 \pm 4.28 \%$ \\
Mediastinum $(n=2)$ & $4.44 \pm 2.26 \%$ \\
All metastatic nodules & $5.62 \pm 1.81 \%$ \\
\hline Serial sections of metastatic nodules were stained for the Y \\
chromosome (FISH) and for cytokeratin. Data are expressed as the \\
number of Y chromosome-positive cells per 100 cytokeratin-positive \\
cells (cancer cells).
\end{tabular}

using MiaPaCa- $2 \pm$ CAhPSCs $^{5}$ and those from the current model using AsPC-1 \pm CAhPSCs indicated increased CD31 staining in primary tumors of mice injected with MiaPaCa-2 and hPSCs compared to MiaPaCa-2 alone tumors, but no change in AsPC-1 + CAhPSC tumors compared to AsPC-1 alone tumors (Table 3).

\section{In Vitro}

Tube formation by HMEC-1 was significantly stimulated by secretions from four different CAhPSC and six different NhPSC preparations (Figure 4A). The inductive effect of CAhPSC secretions on HMEC-1 tube formation was significantly reduced in the presence of anti-human VEGF neutralizing antibody (Figure 4B), suggesting that VEGF may mediate, at least in part, the observed stimulation of angiogenesis by CAhPSCs.

\section{Effect of Cancer Cells on Transendothelial Migration of hPSCS}

Both cancer-associated and normal hPSCs exhibited a basal rate of migration through an endothelial cell monolayer; notably, this migration was significantly increased when hPSCs were cocultured with AsPC-1 cells (Figure $5 A)$. In the presence of anti-human PDGF-BB-neutralizing antibody, CAhPSC-induced transendothelial migration returned to basal levels indicating that PDGF is a major inducer of PSC migration in cancer cell secretions (Figure 5B).

\section{Discussion}

While the aggressiveness of cancer cells themselves is an important feature in cancer metastasis, the role of stromal cells has been increasingly recognized. ${ }^{4,5,8,37,38}$ In our current study, we have not only confirmed the
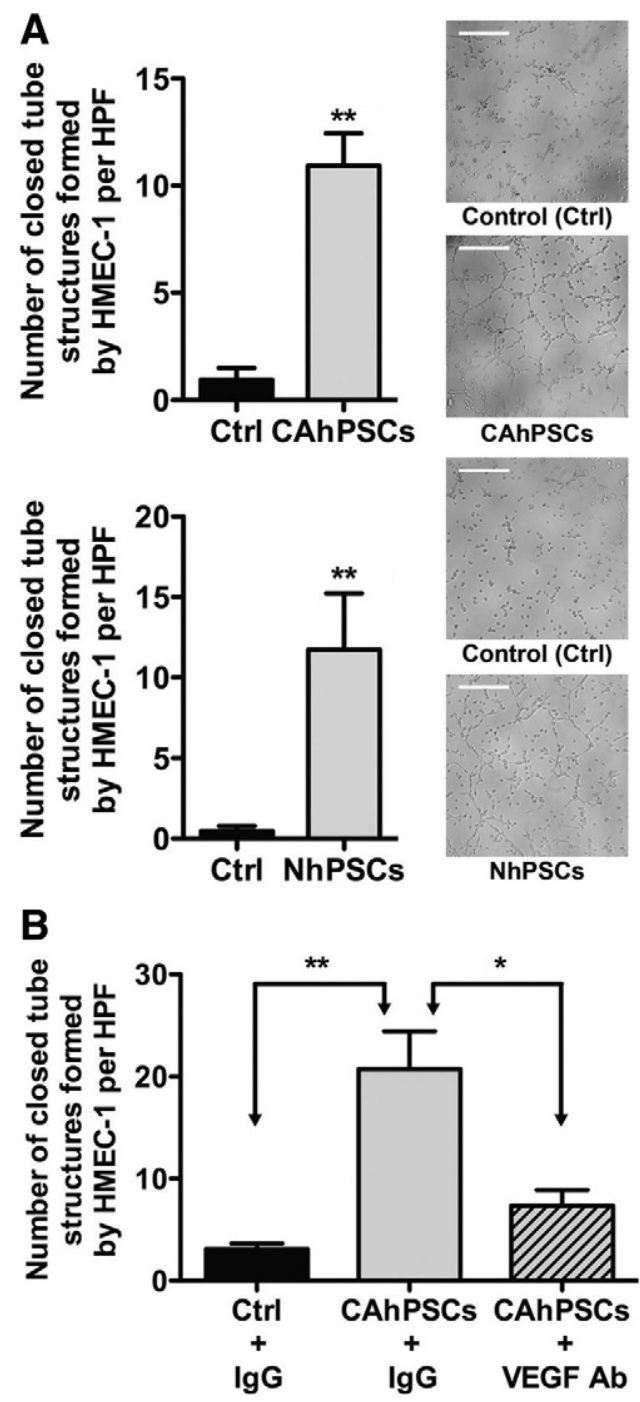

Figure 4. Angiogenesis assay. A: Effect of hPSCs on tube formation by HMEC-1 $(\times 100)$ : Conditioned media from CAhPSCs significantly stimulated tube formation by HMEC- 1 cells $(* * P<0.005 ; n=4$ different CAhPSC preparations; $\mathrm{Ctrl}=$ Control). Similar results were found with conditioned media from NhPSC preparations (** $P<0.01 ; n=6$ different NhPSC preparations). Scale bars $=50 \mu \mathrm{m}$. B: Effect of VEGF-neutralizing antibody on angiogenesis in vitro: Tube formation of HMEC-1 cells was significantly reduced in the presence of CAhPSC secretions pretreated with VEGF-neutralizing antibody $\left({ }^{*} P<0.01,{ }^{*} P<0.005 ; n=3\right.$ different CAhPSC preparations).

facilitatory role of PSCs on local tumor growth and distant spread, but more importantly, we provide unequivocal evidence that hPSCs originating from the primary tumor are present in metastatic nodules. This novel finding indicates that hPSCs (stromal cells) have the ability to

Table 3. CD31 Expression in Primary Tumors

\begin{tabular}{lcc}
\hline Tumor model & $\begin{array}{c}\text { Cancer cell line alone } \\
(\% \text { of total area per HPF) }\end{array}$ & $\begin{array}{c}\text { Cancer cells }+\mathrm{hPSCs} \\
(\% \text { of total area per HPF) }\end{array}$ \\
\hline AsPC-1 model & $3.54 \pm 0.91$ & $3.16 \pm 0.45$ \\
MiaPaCa-2 model & $3.07 \pm 0.53$ & $6.80 \pm 1.09^{*}$ \\
\hline
\end{tabular}

CD31 expression in primary tumors from mice injected with AsPC-1 + hPSCs was similar to that in primary tumors from mice injected with AsPC-1 alone. However, it was significantly increased in primary tumors from mice injected with MiaPaCa-2 + CAhPSCs compared with that in tumors from mice injected with MiaPaCa-2 alone $\left({ }^{\star} P<0.05 ; n=7 /\right.$ group $)$. 
300
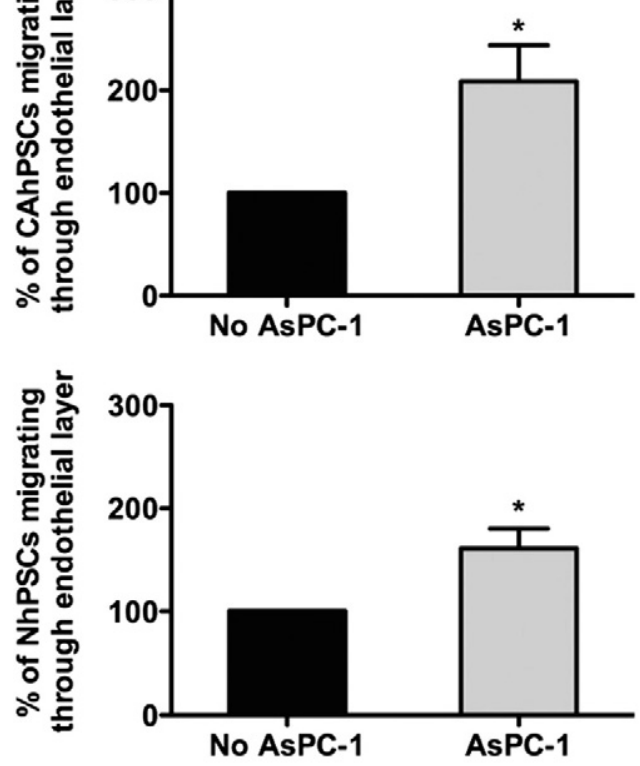

B

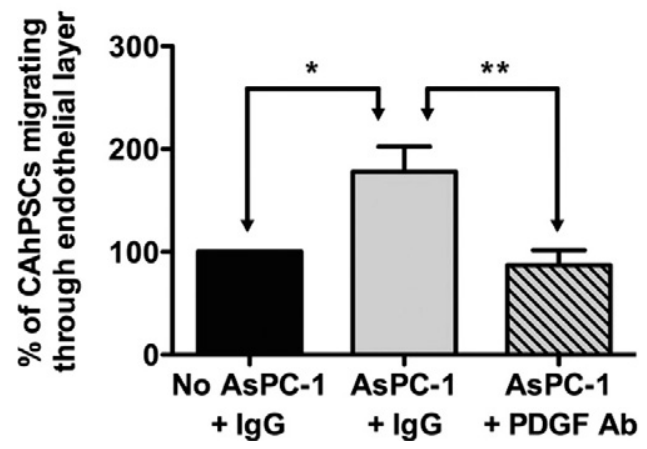

Figure 5. Transendothelial migration of hPSCs. A: The proportion of CAhPSC or NhPSCs migrating through an endothelial cell monolayer was significantly increased in the presence of AsPC- $1{ }^{*} P<0.05 ; n=3$ separate CAhPSC preparations and 5 separate NhPSC preparations). B: Effect of PDGF-neutralizing antibody on transendothelial migration: AsPC-1-stimulated transendothelial migration of CAhPSCs was prevented in the presence of PDGFneutralizing antibody $\left({ }^{*} P<0.05,{ }^{* *} P<0.01 ; n=4\right.$ different CAhPSC preparations).

travel with cancer cells to distant metastatic sites, thereby challenging the concept that migration to distant organs is the sole preserve of cancer cells.

A sex mismatch approach allowed the identification of Y chromosome-positive cells in metastatic nodules at regional and distant metastatic sites in each mouse injected with AsPC-1 + male hPSCs. Dual staining for GFAP (PSC-selective marker) and FISH confirmed that these are the exogenously introduced human PSCs. Furthermore, because these PSCs were found in metastatic sites 6 weeks after they were first introduced into the mouse pancreas, our results support the notion that PSCs are able to i) intravasate/extravasate into and out of vessels, ii) survive in the circulation, and iii) seed in metastatic niches in distant organs. Our findings that hPSCs can migrate through an endothelial cell monolayer, and more importantly, that this migration is induced in the presence of cancer cells further support the concept that hPSCs have the capacity to intravasate/extravasate into and out of blood vessels. We have also shown that the observed inductive effect of cancer cells on transendothelial migration of CAhPSCs is mediated by cancer cellsecreted PDGF, a known chemotactic factor for PSCs. ${ }^{39}$

The observation that PSCs accompany cancer cells on their metastatic journey is not entirely surprising. It is now accepted that the metastatic process involves the detachment of small clusters or aggregates of tumor cells from the primary tumor which then enter the blood or lymphatic circulation. ${ }^{11}$ Because tumor cells and PSCs are closely associated within pancreatic tumors and exhibit strong interactions, it would be reasonable to expect that PSCs form an integral part of the cell clusters detaching from the primary tumor and traveling to distant sites. Another possibility that cannot be discounted is that PSCs travel separately from cancer cells to distant metastatic sites where they establish a microenvironment (the soil) that could facilitate subsequent seeding of cancer cells. Whether the presence of PSCs in metastatic sites is true movement from the primary site or the result of spills during injections may be questioned. However, the latter possibility is unlikely given the facts that i) hPSC containing metastatic nodules were observed at sites left undisturbed during the injections; ii) several metastatic sites per mouse revealed the presence of hPSCs; and iii) every mouse injected with the two cell types exhibited hPSCs in metastases.

Nakagawa et al ${ }^{40}$ have reported that in liver metastases of colon cancer, cancer-associated fibroblasts form a favorable microenvironment for cancer cells, with the view that these cancer-associated stromal cells all originate in the metastatic organ. ${ }^{41}$ However, our findings indicate an additional possibility, that of stromal cells/ PSCs (originating in the primary site) accompanying tumor cells to metastatic sites. Although our observations indicate that tumor cells outnumber human PSCs in metastatic sites, it is worth considering the possibility that small numbers of hPSCs may still be sufficient in the initial stages of metastasis to facilitate seeding and survival of tumor cells in non-primary sites, and to recruit local/host stromal cells which themselves would subsequently facilitate ongoing tumor growth by providing them with extracellular matrix support and by their known effect on increasing cancer cell survival via increased proliferation and reduced apoptosis. ${ }^{4,5}$

With regard to recruitment of host stromal cells in the metastatic site by PSCs originating from the primary tumor, we have shown (using $\alpha$ SMA staining) that there are increased numbers of activated stellate cells in mice receiving AsPC-1 + hPSCs compared to mice receiving AsPC-1 alone. Because the primary antibody for $\alpha \mathrm{SMA}$ used in these studies binds to both human and mouse antigen, the observed increase in activated stellate cell numbers could represent both local proliferation of human PSCs as well as increased recruitment of mouse stromal cells (in this case, hepatic stellate cells) within the 
metastatic site, via the known ability of PSCs to secrete growth factors and cytokines that are chemotactic for stromal cells. ${ }^{42-44}$

In the present study, two different types of human PSCs were used-PSCs isolated by the outgrowth method from resected pancreatic tissue from patients with pancreatic cancer (CAhPSCs) and PSCs isolated by density gradient centrifugation from the histologically normal pancreatic tissue (as confirmed by H\&E staining) from patients undergoing resection for benign pancreatic disorders (NhPSCs). The major difference between these two types of cells is that the former (CAhPSCs) are presumably already influenced (primed) by surrounding cancer cells in vivo and are isolated in their activated phenotype while the latter are obtained from normal pancreas (with no contact with malignant cells) and at the time of isolation are in their quiescent (vitamin A containing) phenotype. Our recent studies indicate that the presumed genetic differences between the two cell types (at isolation) are maintained even after the cells are in culture for a few weeks. Gene microarrays of cultured NhPSCs and CAhPSCs, have revealed 104 dysregulated genes ( $>2$ fold difference, $P<0.05, n=5$ separate preparations of each cell type) between the two cell types. ${ }^{45}$ Yet, when injected into the pancreas of mice with the cancer cells, both CAhPSCs and NhPSCs exerted a significant growth promoting influence on pancreatic tumors when compared to tumors in mice receiving cancer cells alone. It was particularly interesting to find that NhPSCs (presumably free of any prior influence from cancer cells) were able to induce tumor growth and spread in vivo after only 6 weeks of association with the cancer cell line AsPC-1.

Proliferating cancer cells require the establishment of new circulatory beds by angiogenesis for the supply of nutrients essential to their survival. ${ }^{11}$ In the current study, endothelial cell number (as assessed by staining for the endothelial cell marker CD31) was significantly increased within MiaPaCa-2 + hPSC tumors compared to MiaPaCa-2 alone tumors. This finding supports the recently reported increase in CD31 staining in peritumoral stroma of pancreatic cancer in humans, although it is noted that the authors report that the overall microvascular density over the entire tumor section was found to be decreased in human pancreatic cancer sections compared to normal pancreas. ${ }^{3}$ However, we did not observe increased CD31 staining in AsPC-1 + hPSC tumors. This may reflect the fact that AsPC-1 is a highly aggressive cell line, ${ }^{46,47}$ which may itself induce significant angiogenesis, making it difficult to show any further increase in the presence of hPSCs. Our in vivo observations of hPSCinduced tumor angiogenesis in the MiaPaCa-2 + hPSC tumors are supported by our in vitro studies demonstrating that exposure to secretions from CAhPSCs or NhPSCs induces tube formation by HMEC-1 cells. Our study also indicates that the proangiogenic effect of CAhPSCs on HMEC-1 cells is mediated, at least in part, by hPSCsecreted VEGF. Our in vitro findings concur with a recent study by Masamune et $\mathrm{al}^{26}$ reporting increased tube formation of human umbilical vein endothelial cells (HUVECs) exposed to human PSCs cultured under hy- poxic conditions. A study by Erkan and colleagues ${ }^{3}$ has reported increased proliferation of HUVECs in response to PSC secretions under both normoxic and hypoxic conditions, but a decrease in HUVEC proliferation after exposure to cancer cell secretions alone and to secretions obtained from cocultures of cancer cells and PSCs. However, tube formation of HUVECs was not examined in this study. The above studies indicate that the angiogenic process is complex and the response of endothelial cells may vary depending on the prevalent combination of factors in the microenvironment.

In summary, this study has yielded the novel findings that hPSCs (stromal cells) i) accompany cancer cells to distant metastatic sites where they most likely facilitate the seeding, survival, and proliferation of cancer cells; ii) stimulate angiogenesis in pancreatic tumors; and iii) have the capacity to intravasate/extravasate through blood vessels - an ability that is significantly induced by cancer cells. The challenge now is to characterize the pathways responsible for mediating the observed interactions of cancer cells and stromal cells. Our findings indicate that VEGF and PDGF may be of interest in this regard. We were also interested to read a very recent report by Olive et $\mathrm{a}^{48}$ noting that targeting of the Hedgehog pathway to disrupt the stroma in mouse pancreatic cancer improved the delivery and antitumor efficacy of the chemotherapeutic agent gemcitabine. Other areas worthy of investigation (possibly via genomic and proteomic approaches) in this field are the differences in the functions of diseaseassociated (both inflammation-associated and cancerassociated) and normal PSCs, to clarify the processes involved in the evolution of PSCs from a normal to a malignant phenotype.

Progress in pancreatic cancer therapy has continued to be slow and limited, ${ }^{49}$ with most efforts being directed at the cancer cell as a target. The identification of the active role of the pancreatic stroma now provides an alternative strategy to enhance outcomes.

\section{Acknowledgment}

We are grateful to Dr. Nicodemus Tedla (UNSW) for expert advice on FISH methodology.

\section{References}

1. Apte MV, Park S, Phillips PA, Santucci N, Goldstein D, Kumar RK, Ramm GA, Buchler M, Friess H, McCarroll JA, Keogh G, Merrett N, Pirola R, Wilson JS: Desmoplastic reaction in pancreatic cancer: role of pancreatic stellate cells. Pancreas 2004, 29:179-187

2. Bachem MG, Schunemann M, Ramadani M, Siech M, Beger $H$, Buck A, Zhou S, Schmid-Kotsas A, Adler G: Pancreatic carcinoma cells induce fibrosis by stimulating proliferation and matrix synthesis of stellate cells. Gastroenterology 2005, 128:907-921

3. Erkan M, Reiser-Erkan C, Michalski CW, Deucker S, Sauliunaite D, Streit S, Esposito I, Friess H, Kleeff J: Cancer-stellate cell interactions perpetuate the hypoxia-fibrosis cycle in pancreatic ductal adenocarcinoma. Neoplasia 2009, 11:497-508

4. Hwang RF, Moore T, Arumugam T, Ramachandran V, Amos KD Rivera A, Ji B, Evans DB, Logsdon CD: Cancer-associated stromal fibroblasts promote pancreatic tumor progression. Cancer Res 2008 , 68:918-926 
5. Vonlaufen A, Joshi S, Qu C, Phillips PA, Xu Z, Parker NR, Toi CS, Pirola RC, Wilson JS, Goldstein D, Apte MV: Pancreatic stellate cells: partners in crime with pancreatic cancer cells. Cancer Res 2008, 68:2085-2093

6. Apte MV, Pirola RC, Wilson JS: Battle-scarred pancreas: role of alcohol and pancreatic stellate cells in pancreatic fibrosis. J Gastroenterol Hepatol 2006, 21 Suppl 3:S97-S101

7. McCarroll JA, Phillips PA, Santucci N, Pirola RC, Wilson JS, Apte MV: Vitamin A inhibits pancreatic stellate cell activation: implications for treatment of pancreatic fibrosis. Gut 2006, 55:79-89

8. Vonlaufen A, Phillips PA, Xu Z, Goldstein D, Pirola RC, Wilson JS, Apte MV: Pancreatic stellate cells and pancreatic cancer cells: an unholy alliance. Cancer Res 2008, 68:7707-7710

9. Jemal A, Siegel R, Ward E, Hao Y, Xu J, Murray T, Thun MJ: Cancer statistics, 2008. CA Cancer J Clin 2008, 58:71-96

10. Grzesiak JJ, Ho JC, Moossa AR, Bouvet M: The integrin-extracellular matrix axis in pancreatic cancer. Pancreas 2007, 35:293-301

11. Langley RR, Fidler IJ: Tumor cell-organ microenvironment interactions in the pathogenesis of cancer metastasis. Endocr Rev 2007, 28:297-321

12. Klein CA, Blankenstein TJ, Schmidt-Kittler O, Petronio M, Polzer B, Stoecklein NH, Riethmuller G: Genetic heterogeneity of single disseminated tumour cells in minimal residual cancer. Lancet 2002, 360:683-689

13. Liotta LA, Kleinerman J, Saidel GM: Quantitative relationships of intravascular tumor cells, tumor vessels, and pulmonary metastases following tumor implantation. Cancer Res 1974, 34:997-1004

14. Thorban S, Roder JD, Siewert JR: Detection of micrometastasis in bone marrow of pancreatic cancer patients. Ann Oncol 1999, 10 (Suppl 4):111-113

15. Z'Graggen K, Centeno BA, Fernandez-del Castillo C, Jimenez RE, Werner J, Warshaw AL: Biological implications of tumor cells in blood and bone marrow of pancreatic cancer patients. Surgery 2001, 129:537-546

16. Carmeliet P: VEGF as a key mediator of angiogenesis in cancer. Oncology 2005, 69 (Suppl 3):4-10

17. Kerbel RS: Tumor angiogenesis. N Engl J Med 2008, 358:2039-2049

18. Duncan TJ, Al-Attar A, Rolland P, Scott IV, Deen S, Liu DT, Spendlove I, Durrant LG: Vascular endothelial growth factor expression in ovarian cancer: a model for targeted use of novel therapies? Clin Cancer Res 2008, 14:3030-3035

19. Ferrara N: VEGF as a therapeutic target in cancer. Oncology 2005,69 (Suppl 3):11-16

20. Kanda M, Nomoto S, Nishikawa Y, Sugimoto H, Kanazumi N, Takeda $\mathrm{S}$, Nakao A: Correlations of the expression of vascular endothelial growth factor B and its isoforms in hepatocellular carcinoma with clinico-pathological parameters. J Surg Oncol 2008, 98:190-196

21. Keedy VL, Sandler AB: Inhibition of angiogenesis in the treatment of non-small cell lung cancer. Cancer Sci 2007, 98:1825-1830

22. Nayha $V$, Stenback F: Angiogenesis and expression of angiogenic agents in uterine and ovarian carcinosarcomas. APMIS 2008, 116:107-117

23. Peyromaure M, Badoual C, Camparo P, Grabar S, Goulvestre C, Fulla $\mathrm{Y}$, Vieillefond A, Mao K, Dinh-Xuan AT: Plasma levels and expression of vascular endothelial growth factor-A in human localized prostate cancer. Oncol Rep 2007, 18:145-149

24. Seo Y, Baba H, Fukuda T, Takashima M, Sugimachi K: High expression of vascular endothelial growth factor is associated with liver metastasis and a poor prognosis for patients with ductal pancreatic adenocarcinoma. Cancer 2000, 88:2239-2245

25. Toi M, Inada K, Suzuki H, Tominaga T: Tumor angiogenesis in breast cancer: its importance as a prognostic indicator and the association with vascular endothelial growth factor expression. Breast Cancer Res Treat 1995, 36:193-204

26. Masamune A, Kikuta K, Watanabe T, Satoh K, Hirota M, Shimosegawa T: Hypoxia stimulates pancreatic stellate cells to induce fibrosis and angiogenesis in pancreatic cancer. Am J Physiol Gastrointest Liver Physiol 2008, 295:G709-G717

27. Mahadevan D, Von Hoff DD: Tumor-stroma interactions in pancreatic ductal adenocarcinoma. Mol Cancer Ther 2007, 6:1186-1197

28. Ide T, Kitajima Y, Miyoshi A, Ohtsuka T, Mitsuno M, Ohtaka K, Miyazaki K: The hypoxic environment in tumor-stromal cells acceler- ates pancreatic cancer progression via the activation of paracrine hepatocyte growth factor/c-Met signaling. Ann Surg Oncol 2007, 14:2600-2607

29. Yamanaka $Y$, Friess $H$, Buchler M, Beger HG, Uchida E, Onda M, Kobrin MS, Korc M: Overexpression of acidic and basic fibroblast growth factors in human pancreatic cancer correlates with advanced tumor stage. Cancer Res 1993, 53:5289-5296

30. Ades EW, Candal FJ, Swerlick RA, George VG, Summers S, Bosse DC, Lawley TJ: HMEC-1: establishment of an immortalized human microvascular endothelial cell line. J Invest Dermatol 1992, 99:683-690

31. Bachem MG, Schneider E, Gross H, Weidenbach H, Schmid RM, Menke A, Siech M, Beger H, Grunert A, Adler G: Identification, culture, and characterization of pancreatic stellate cells in rats and humans. Gastroenterology 1998, 115:421-432

32. Apte MV, Haber PS, Applegate TL, Norton ID, McCaughan GW, Korsten MA, Pirola RC, Wilson JS: Periacinar stellate shaped cells in rat pancreas: identification, isolation, and culture. Gut 1998, 43:128-133

33. Bodnar RJ, Yates CC, Wells A: IP-10 blocks vascular endothelial growth factor-induced endothelial cell motility and tube formation via inhibition of calpain. Circ Res 2006, 98:617-625

34. Marchetti M, Vignoli A, Russo L, Balducci D, Pagnoncelli M, Barbui T, Falanga A: Endothelial capillary tube formation and cell proliferation induced by tumor cells are affected by low molecular weight heparins and unfractionated heparin. Thromb Res 2008, 121:637-645

35. Ockaili R, Natarajan R, Salloum F, Fisher BJ, Jones D, Fowler AA 3rd, Kukreja RC: HIF-1 activation attenuates postischemic myocardial injury: role for heme oxygenase-1 in modulating microvascular chemokine generation. Am J Physiol Heart Circ Physiol 2005 289:H542-H548

36. Snedecor GW, Cochran WG: Statistical Methods. ed 8. Ames (IO) lowa State University Press, 1989, pp 1-456

37. Inafuku Y, Furuhata T, Tayama M, Okita K, Nishidate T, Mizuguchi T, Kimura Y, Hirata K: Matrix metalloproteinase-2 expression in stromal tissues is a consistent prognostic factor in stage II colon cancer. Cancer Sci 2009, 100:852-858

38. Sloan EK, Ciocca DR, Pouliot N, Natoli A, Restall C, Henderson MA, Fanelli MA, Cuello-Carrion FD, Gago FE, Anderson RL: Stromal cell expression of caveolin-1 predicts outcome in breast cancer. Am J Pathol 2009, 174:2035-2043

39. Phillips PA, Wu MJ, Kumar RK, Doherty E, McCarroll JA, Park S, Pirola RC, Wilson JS, Apte MV: Cell migration: a novel aspect of pancreatic stellate cell biology. Gut 2003, 52:677-682

40. Nakagawa H, Liyanarachchi S, Davuluri RV, Auer H, Martin EW Jr, de la Chapelle A, Frankel WL: Role of cancer-associated stromal fibroblasts in metastatic colon cancer to the liver and their expression profiles. Oncogene 2004, 23:7366-7377

41. Mueller L, Goumas FA, Affeldt M, Sandtner S, Gehling UM, Brilloff S, Walter J, Karnatz N, Lamszus K, Rogiers X, Broering DC: Stromal fibroblasts in colorectal liver metastases originate from resident fibroblasts and generate an inflammatory microenvironment. Am J Pathol 2007, 171:1608-1618

42. Karger A, Fitzner B, Brock P, Sparmann G, Emmrich J, Liebe S, Jaster R: Molecular insights into connective tissue growth factor action in rat pancreatic stellate cells. Cell Signal 2008, 20:1865-1872

43. Shek FW, Benyon RC, Walker FM, McCrudden PR, Pender SL, Williams EJ, Johnson PA, Johnson CD, Bateman AC, Fine DR, Iredale JP: Expression of transforming growth factor-beta 1 by pancreatic stellate cells and its implications for matrix secretion and turnover in chronic pancreatitis. Am J Pathol 2002, 160:1787-1798

44. Yang C, Zeisberg M, Mosterman B, Sudhakar A, Yerramalla U, Holthaus K, Xu L, Eng F, Afdhal N, Kalluri R: Liver fibrosis: insights into migration of hepatic stellate cells in response to extracellular matrix and growth factors. Gastroenterology 2003, 124:147-159

45. Phillips PA, Chow E, Kaplan W, Cowley M, Vonlaufen A, Yang L, Xu Z, Biankin AV, Goldstein D, Pirola RC, Wilson JS, Apte MV: Gene expression profiling of human pancreatic stellate cells and their roles in pancreatic cancer progression. Pancreatology 2009, 9:488

46. Chen WH, Horoszewicz JS, Leong SS, Shimano T, Penetrante R, Sanders WH, Berjian R, Douglass HO, Martin EW, Chu TM: Human pancreatic adenocarcinoma: in vitro and in vivo morphology of a new tumor line established from ascites. In Vitro 1982, 18:24-34 
47. Tan MH, Chu TM: Characterization of the tumorigenic and metastatic properties of a human pancreatic tumor cell line (AsPC-1) implanted orthotopically into nude mice. Tumour Biol 1985, 6:89-98

48. Olive KP, Jacobetz MA, Davidson CJ, Gopinathan A, McIntyre D, Honess D, Madhu B, Goldgraben MA, Caldwell ME, Allard D, Frese KK, Denicola G, Feig C, Combs C, Winter SP, Ireland-Zecchini H, Reichelt S, Howat WJ, Chang A, Dhara M, Wang L, Ruckert F, Grutzmann R, Pilarsky C, Izeradjene K, Hingorani SR, Huang P, Davies SE, Plunkett W, Egorin M, Hruban RH, Whitebread N, McGovern K, Adams J, lacobuzio-Donahue C, Griffiths J, Tuveson
DA: Inhibition of Hedgehog signaling enhances delivery of chemotherapy in a mouse model of pancreatic cancer. Science 2009, 324:1457-1461

49. Poplin E, Feng Y, Berlin J, Rothenberg ML, Hochster H, Mitchell E, Alberts S, O'Dwyer P, Haller D, Catalano P, Cella D, Benson AB 3rd: Phase III, Randomized Study of Gemcitabine and Oxaliplatin Versus Gemcitabine (fixed-dose rate infusion) Compared With Gemcitabine (30-minute infusion) in Patients With Pancreatic Carcinoma E6201: A Trial of the Eastern Cooperative Oncology Group. J Clin Oncol 2009, 27:3778-3785 